\title{
Index
}

Aberdeen Palais 92

Abravanel, Genevieve 7, 20

Abreu, Christina D. 170, 175n.28

Adair, Enid 51

Adorno, Theodor 10

advertising 11, 27, 48, 53, 56, 115, 159, 160-3, 164-5, 194, 198, $201,219,246,260-1$

Africa 8, 153, 156

influence on dancing 8, 24, 151, 159

influence on music 22-3, 28

African Americans 28, 136

influence on dancing 21, 22-3, 25, $27,65,67,84,151-4,170-3$, 183, 197, 203-4, 242-3, 249-52, 254

GIs in Britain 231, 232-6, 250

air raids 191, 209-10, 212-13, 215, 217, 219

'Alexander's Ragtime Band' 23, 184

Allen, Maud 20, 131

All-Britain Jitterbug Championship 248

All-England Jitterbug Championship 243

Americanisation 2, 3, 4, 5, 8, 12, 14-15, 17, 42-3, 159-65, 170-3, 180, 184-5, 192, 197, 202, 203-4, 241-2, 248-50, 257, 263, 264

see also African Americans; United States

American military 21, 28

in Britain during Second World War 15, 208, 227-8, 232-6, 241, 242, 243, 244, 247, 250, 257
American Red Cross clubs 227

'Ampstead way 261

Anglican Church 230

Anglicisation 4, 5, 8-9, 14-15, 142-3, 150-4, 160-3, 164-5, 179-80, 196-8, 201-2, 241, 248-50, 251, 254

animal dances 23, 24, 150, 257

Argentina 8, 12, 20, 21, 24, 84

A Rising Wind 236

Armstrong, Louis 28

Arthur Murray dance studios 182, 258

Association of Ballrooms 214

Astoria dance hall 57, 92, 101, 134, 213-14, 215-16, 219-20

Aspin dance hall (Bolton) 126, 195-6

Australia 51, 84, 156, 157, 159, 258, 259

Asia 156, 158

influence on dance culture 80, 83-4

see also Orientalism

Astaire, Adele 169

Astaire, Fred 169-70, 172

Auxiliary Territorial Service 228-9

Bachelor Mother 243

Bailey, Peter 10, 164, 224

ballet 32, 46, 50, 52

Barnett, Albert 161-2

Barrowlands ballroom (Glasgow) 223

BBC 100-1, 181, 193, 217

see also radio

BBC Dancing Club 55, 56, 258, 260

Baxter, A. Beverley 109 
Bechet, Sydney 28

Belfast 145

benefits of dancing 12, 35-8, 257

to beauty 36-7

to health $35-6,60,118-19$

Berlin, Irving 23, 184

Big Apple (dance) 171, 183, 242

The Big Store 243

Birmingham 58, 83, 87, 93, 214

black bottom 72

blackface minstrelsy $8,23,67,152$

black-out 210-11, 214, 216, 219

black-out stroll 195, 219-20, 226

Blackpool 57, 79, 86, 93, 188, 221, 249

dance championship 52, 157, 201

Bland, Lucy 6, 112

Blitz 191, 208, 212, 215, 216

Blitz spirit and dancing 4, 15, 191-2, 208, 210-13, 215, 225, 253

blues (dance) 45, 49, 62, 65, 91, 95-6, 152

blues (music) 65

Bolton 89, 123, 126, 146-7, 199, 201, 216, 244

boomps-a-daisy 179, 201

Boston (dance) 7, 22, 24, 26, 52, 53

Bracken, Brendan 234

Bradford 87

Bradley, Josephine 55, 66, 69, 95, $155-6,166,171$

Bramall, Nancy 148, 154

Brewster's Millions 169

Briggs, Asa 100

Brighton 58, 79, 83, 93, 214, 247

Bright Young Things 117

Britain under Shellfire 213

British Empire Exhibition 84

Buckland, Theresa Jill 7, 150

bunny hug 23

Bus, Thomas 155, 166

cabaret $51,68,85,92,97,99$

Café de Paris 79, 86, 210, 215, 219, 226

cakewalk 23, 181

Canada 84, 96, 159
Canadian military 227, 234, 243, 244, 247

Canterbury 94

caranga $169-70$

Cardiff 93, 145

Caribbean 8, 28, 151, 153, 227, 231

carioca 99, 170-1

Carr, Michael 194

Casani, Santos 10, 35-6, 37, 49, 51, $53,54,55,63,68,117,118-19$, 150,158

Casino dance hall (Warrington) 234-5

Castle, Irene 26-7, 28, 142, 151, 153-4, 169

Castle, Vernon 24, 26-7, 28, 142, 151, 153, 169

Celtic nationalism 5

Ceylon 259

Chalmers, Patrick 70, 131

Channel Islands 222

Chaperlin, Charles 98

Charleston (dance) 4, 38, 45, 62, 66-70, 71, 97, 106, 114, 150, 151, 152-3, 162, 165, 172, 179, $182,210,244,250,257,264$

flat progressive Charleston 67-8, 70, 152, 249

Charleston Ball 68

cherry hop 179

Chestnut Tree 14, 179, 190, 192-3, 194, 196, 197-9, 201-3, 220

Chicago 28

Chilo, Nora 160-1

China 159

Chinese 136, 153

Christie, Agatha 127

church dances 230

cinema 11, 86-7, 102, 164, 172, 189, $210,214,243,264$

Cinematograph Films Act (1927) 164

circus 224

civil defence 218

Clarke, Phyllis 55

class $3,4,6,9,10,12,39,49,58,107$, $114,116-17,126,127,134,136$, $145,153,178,209,212,225-7$, 228 
dancing and social unity 15,19 , 33-4, 143, 144, 145, 189-90, $192,208,211,219,225-7,253-4$ see also dance halls and class cockneys 178, 181, 185-6, 189-90, 191, 261

college swing 150

Colonial Office 234, 235

colonial subjects in Britain 225-6, 227, 231-6

colour bars 231-2, 233-6, 250

Come Dancing 258, 264

commercial nationalism 4-5, 10, $14-15,145,160-5,180,183-6$, 193, 196-8, 201-4, 209, 218-25, $248-9,261$

concert dance 20, 83, 131, 143

Connor, Tommy 193, 197, 220

continental (dance) 169

Constantine, Learie 231-2

Cook, James W. 10

Coward, Noel 106

Cricklewood dance hall 59, 94

crime 13, 116, 122, 123-5,

Criminal Law Amendment Act (1885) 132

criticisms of dancing 2-3, 37-8, 79, 142,145

Edwardian period 25-6

France 47

interwar period $12,29-35,47-8$, 66-7, 107-14

Second World War 221-2, 244-8, 249, 252

post-1945 257, 261-3

see also racism and dancing; respectability

Crombie, Anthony 210, 215, 216, 253

Cuba 168-9, 170

culture industry 10-11, 18n.20

Cunningham, George 161

Daily Express 107-9

Daily Sketch 51-2, 100

Danceland Publications 87, 99, 222-4, 249

dance bands 4, 11, 62, 63, 65, 70, 71, $81,85,87,91,92,96,97,98$,
100-1, 114, 164, 166, 184, 210,

217, 226, 242, 262

see also musicians

dance clubs 23, 27, 78-9, 95

dance competitions $1,10,12,45,51-2$, 56-8, 61, 64, 68, 71, 79, 96, 128, $146,157,168,225,243,245$, 258-9, 260, 263

dance craze

after First World War 2, 12, 19-20, 21, 33, 38-9, 45, 46, 55, 77, 79, $112,208,210,253$

during Second World War 208-9, 210-17, 223, 233-6

dance hall industry 3-6, 7, 9-16, 30, $39,50,71,77,84,89,90,93$, 95-9, 107, 110, 128, 169, 195, 214, 217-25, 233-6, 241, 243, $244,246,250,254,257,262-3$

and dancing public $88,90-102$, 170-3, 180, 186-9, 191-2, 197-9, 201-4, 218-19, 246

origins $78-81,85-8$

see also dance halls; Mecca Circuit dance halls 2, 7, 19, 23, 45, 50, 51, 57, $58,59,64,66-7,71,73,77$, 106-7, 119, 160, 166, 168, 178, 181, 186, 193, 200, 226, 243-5, $258,260,261,263,264$

and class 77-9, 80, 87, 93-5, 114, $121-2,122-3$

décor $81-5$

during Second World War 209-10, 210-14, 215-16, 217-19

experience of 85, 89, 90-9, 100-2, 120

see also dance hall industry; Mecca Circuit; respectability and dance halls; sexuality and public dancing spaces

dance instruction 5, 10, 32, 33, 44, $46-7,53,56,57,58-60,61,63$, 71-2, 96, 115-16, 128, 158, 163, $183,258,259,263$

see also dance manuals; dancing schools dance manuals 53-4, 55, 56, 58, 59, 61, 72, 114, 118-19, 156, 158, $162,165,260,264$ 
dance marathons 243

dance music 36, 70-1, 91, 100, 161, $166-7,253$

strict tempo 55, 91, 166-7, 249, 258,262

dance partners $10,13,50,55,81-2$, $87,107,116,127-37$

dance print culture $7,10-11,12,36$, $45,53,56,58,61,69,87$, 99-100, 114, 161-3, 169, 170, 216, 221-25, 243, 246, 264

dance profession 1, 2, 3-6, 7, 9-16, 30, 39, 70-3, 94, 95, 96, 118, 125, 128, 134-5, 142-63, 164, 166-9, 170-3, 184, 197, 199-201, 217, 241, 244, 246, 247, 249-50, 254, 257, 258-60, 263

and dancing public 56-61, 61-73, $164-6,168,172-3,260-3$

defences of dancing 35-9, 47 financial concerns $3,5,10,38,39$, $45,47-8,50,51,60,61,63,69$, $71-3,167,200-1,217,218$ professionalisation 12-13, 44-56, 107

professional organisations $52-3$ transmission of dances $1,2,21,24$, $61,72,163,165$

see also informal conferences; standardisation

dance promoters 29, 94, 126, dance teachers see dance instruction; dance profession

Dancer's Victory Fund 217

dances (other venues) 11, 29-30, 77, 78-9, 93-4, 121, 199, 214, 215

dancing public 3-6, 9-16, 19, 30, 45, $52,53,56,58-9,60-1,64,66$, 69, 70, 72, 77-8, 88, 89, 90-102, 146-7,160-1, 166, 167, 170, 180, $183,195,236,241,244,246$, 247-8, 250, 257, 260-1

see also dance hall industry and dancing public; dance profession and dancing public

dancing schools $1,3,7,10,12,19,21$, $25,27,38,45,46,49-50,56,65$,
66, 69, 71, 72, 79, 87, 93, 114, $117,142,156,158,165,167-8$, $182,201,219,258,263,264$

Dancing Times 21, 46, 49, 53, 56, 58, 99, 115, 125, 153, 158, 162, 187

Dancing with the Stars 264

Daunton, Martin 30

Davies, Andrew 121, 124

Davies, Byron 193, 196

Davis, R.T. 242-3, 245

Davson, Harry 161

Deansgate 123

DeGrazia, Victoria 8

Desmond, Jane 9

diplomacy 7, 179, 182, 190, 264

disco 15, 263

discotheques 263

Duggan, Tommy 194

Dunkirk 215

Defence of the Realm Act (1914) 28

democracy

expressed through dancing 4, 5, 20, 33-4, 180, 190-1, 192, 197, 198, 208, 209, 222-5, 236, 253-4, 264

demonstrations see exhibition dancing Denmark 52, 86, 155, 157, 158, 259

department stores 24, 51, 83, 182

Depression 71, 153, 167, 243, 253-4

Dilworth, Charles 130

dog trot 23

Douglas (Isle of Man) 126, 147

Dover 213, 215, 226

Duncan, Isadora 20

Edinburgh 87, 88, 93, 132-3, 145

Edinburgh Palais 88, 122

elite society $24,78-9,116-17,178$

Ellington, Duke 92

Empire 5, 6, 84, 153, 158-9, 173, 182, 202, 234, 250

dancing and imperial unity 15 , 225-6, 227, 234-6, 250

imperialism and dancing 84-5 see also colonial troops in Britain

Empress Ballroom 79, 157, 221

Empress Rooms 55, 131 
England, Adele 87, 170, 181, 182, 185-6, 188, 193, 194-6, 198, 200, 221, 248, 261

English style of ballroom dancing 4, 5, $8,12-13,45,57,60-1,62,64$, 69, 70, 71, 72, 85, 91, 142-60, $165,167,173,180,182,197$, 202, 246, 247, 248, 253, 257, 258-61, 262-4

and gender 49, 150

global dissemination 14, 143, 144, 156-60, 162, 258-9, 264

and race 49, 143, 151-4

see also Anglicisation; dancing and national characteristics; informal conferences; standardisation

Europe 6, 8, 25, 26, 28, 47, 49, 57, 79, $84,96,100,117,143,150,154$, 155, 156, 157-9, 162, 166, 181-2, 190, 215, 222, 258-9

Continental influence on dancing 1 , 7-8, 12, 21, 22, 24, 65, 83, 84, 151,159

Europe, James Reese 27, 28

evacuation 191, 220, 225, 226

exhibition dancing 1, 10, 12, 21, 24, 27, 45, 50-1, 56-8, 61, 64, 69, $85,92,96,97-9,146,201$, 242-3, 244, 246, 250-1, 258, 260

Fairley, Alan 87, 125-6, 234

femininity see women; women and dancing

Farjeon, J. Jefferson 106

fashion $24,27,67,83,114,115,117$, 201

fiction $11,127,135-6$

film $4,7,8,21,51,56,71,73,78,83$, $102,143,160,164,168-70$, 178-9, 182, 191, 243-4, 258

First World War 6, 12, 19, 21, 27-8, 78, 95, 131, 157

impact on dancing 30, 32-5

and gender relations 34-5, 107-10, 114,120

see also dance craze

five-step 14, 72, 143, 161, 163, 165, 198 flapper

see modern woman

flapper vote 120

Flying Down to Rio 169

folk dancing 31-2

Fontana, Georges 57, 161

Fontana, Marjorie 57, 161

football 4

Fox, Harry 27

foxtrot $1,2,4,8,21,27-8,29,45,46$, $48,49,51,62-3,65,70-1,85$, 91, 95-6, 98, 148, 150, 151, 152, 157, 158, 168, 172, 179, 194, 198, 199, 242, 247, 250, 253, 254, 262

see also standard four

France 1, 2, 28, 47, 84, 155, 157, 213

see also Paris

Franks, A.H. 200

French soldiers 28, 227

Funny Face 169

Furber, Douglas 185

Gas Mask Balls 217

gazook 179

gender 3, 4, 6, 7, 9, 10, 12, 39, 107, $127,136,145,209,225-6,231$

see also English style of ballroom dancing and gender; First World War and gender relations; men and dancing; women; women and dancing

generation 22, 26, 31, 93, 97, 145, $182,198,226,242,252,254$

Gerardo 51

German offensive (1940) 208, 216, 221

Germany 155, 157, 158, 190, 216, $222,235,254,259$

Gillespie, John 200

Glasgow 51, 58, 82, 84, 87, 93, 134, 169,223

government 11, 209, 210, 227, 231, 232, 234, 236

Grable, Betty 150

Grafton Galleries 48, 79

Grafton Rooms (Liverpool) 151, 208, 214, 232

Graham, Martha 20 
gramophone 28, 94, 100, 111, 193, 264

Gray, Captain J.S. 57, 90-1, 94, 214, 226, 244

Great Migration 28

grizzly bear 23

Grosvenor House 210, 216

Guy, Stephen 189

Hall, Henry 101, 102

Hammersmith Palais 2, 11, 15-16, 29, $57,62,77,78,79-80,83,90,94$, $116,121,210,211,217,223$

Handsome Territorial 14, 179, 194-5, 196, 197-8, 202-3

Hampstead Heath 186, 187, 261

Harding, Belle 49, 55,

Harlem 84, 171, 182, 242

Harrisson, Tom 86, 89, 184, 187, 190, 202, 216

heebie jeebies 72

Heimann, Carl L. 13, 14, 86-7, 125-6, 178-9, 180-1, 184, 190-2, 193, 195, 198, 201, 234, 241, 246, 248-9

see also Mecca circuit

hesitation waltz 22, 48, 52

A History of English Ballroom Dancing 21, 144

Hitler, Adolf 190, 191, 222-4, 225

holiday camps 258

Home Guard 218

Home Office 214, 216, 232, 233

hooliganism 124, 125-6

horse racing 224

Horwood, Catherine 117

hotel dancing 94-5, 121

Hobsbawm, Eric 92, 170

Hughes, Alec 89, 90, 91, 92, 96-7, 98-9, 194-5, 203, 220, 244

Hughes, Leonora 48

Humphreys, Lisle 55

Hurley, Alec 185, 186

Hylton, Jack 102, 161

Immoral Traffic (Scotland) Act (1902) 132

Imperial Society of Teachers of Dancing $52,55,77,148,154,187$
India 84,159, 259

informal conferences 45, 48-9, 53, 61, $142,153,163$

Great Conference 60, 61, 69, 71, 72, $135,159,163$

International Council of Ballroom Dancing 259

international style 159, 263

Italy 190, 220

Irish home rule 5

Japan $157-8$

jazz 7, 8, 21, 28-9, 31, 37, 38, 46, 78, $80,91,92,96,111,136,147$, $151,152,153,158,165,166-7$, $170,183,254$

see also swing music

Jazz Age 1, 8, 20, 21, 151, 159, 264

jazz roll 29

Jehan, Delicia Husna 261

Jews 92, 136

jitterbug 4, 8, 10, 15, 38, 98, 150, 151, 173, 203-4, 228, 233, 241-54, 257, 262

refined jitterbug 248-50, 254

jive 12, 249-50, 254, 257, 260, 261-3

Jones, Gareth Stedman 190

Joplin, Scott 23

juke joints 23

Kato, James Hyojiro 157-8

Kelley, Robin D.G. 8

Kennedy, Jimmy 193, 194

Kilpatrick, Pat 55, 259

Knees Up, Mother Brown 14, 179, 195-6, 197, 202

Kosmo Club trial 132-3, 134

Labour Party 179, 204n.7

Lambeth 178, 184, 186, 187

Lambeth Walk (dance) 8, 14-15, 100, 173, 178-92, 193, 194, 195, 196, 197, 200, 201-3, 219, 221, 222, $242,246,248,261,264$

and class 179, 180, 186, 189-90, 192

as national dance 179-80, 184-92, 198 
Lambeth Walk (film) 178-9, 204n.5

Lambeth Walk (song) 100, 181, 182, 185, 190

'Lambeth Walk - Nazi Style' 191

lancers $21,31,181,261$

Lane, Lupino 178-9, 181, 185, 186, $187,189,191$

Langhamer, Claire 6, 114

Langdon, Claude 121, 125

Lennard, Jose 51, 55, 68

Latin America 6, 8, 84

influence on dancing $8,12,20,21$, 24, 84, 143-4, 149-50, 151, 159, 167-70, 264

Lavelle, Doris 201

Leeds 33, 46, 87, 217, 245

Leslie, Murray 111

Levey, Ethel 31

Leyton 29-30

licensing 122, 124

Lidgett, Scott 230

Lido dance hall (Croydon) 86

Light, Alison 189

Lindy hop 171, 242, 249

Little Englandism 5, 196-7, 202

Liverpool 27, 58, 93, 146, 147, 151, 208, 214, 232, 233

Liverpool Echo 100

Llandudno 226

Locarno dance hall (Glasgow) 87

Locarno dance hall (Streatham) 81, 86, $87,90,91,97,98,123,131,134$, 181, 187, 193, 194-5, 202, 203, 214, 218, 246, 251

Locarno dancing school 87, 181

London 2, 19-20, 21, 22, 27, 36, 48, $51,53,56,57,59,66,67,77,78$, $80,82,83,89,93,95,122,130$, $136,142,145-7,148,150,152$, 156, 163, 169, 172, 178, 180, 183, 186, 187, 188, 194, 211, 212, 225, 226, 229, 231, 243, 244, 245, 258, 261

Lord Haw-Haw 223

Lord Trenchard 229

Los Angeles 159

Loss, Joe 101, 210, 216, 219, 244

Lunn, Kenneth 5

Lyons, Betty 210
Mackenzie, Alec 1, 2, 8, 159, 163

Mackenzie, James 224, 250, 251

Mackenzie, Maud 159, 163

Madge, Charles 89, 187

Malnig, Julie 9, 112

Manchester 93, 121, 124, 146, 147, 171,211

Mandler, Peter 5, 149

marketing 14-15, 24, 71, 87-8, 97-8, $110,160-5,180,183-4,190-1$, 193-6, 198, 218-225, 246, 248

Marx Brothers 243

masculinity 4, 107, 136, 150

see also First World War and gender relations; men and dancing

Mass Observation 11, 13, 38, 58, 77, $86,87,88,89,90-7,99,100$, 121-3, 126-7 128, 129, 130,134, $146,147,148,156,167,169$, 170-2, 178, 181, 184, 185-6, 187-8, 190-1, 194-6, 199-203, $213,214,215,216,218,220$, $243,244-5,250-1$

master of ceremonies 81, 126, 221, 245

McCardie, Justice 132, 133, 134

McKibbin, Ross 114, 122

McMains, Juliet 47, 147, 263

Me and My Girl 178, 179, 180, 189, 204n.2, 204n.5

Mecca circuit 11, 13, 77, 86-8, 89, 90, 91, 93, 96, 99, 125-6, 127, 128, 134, 170, 178-80, 180-1, 183-6, 187, 190-2, 192-9, 217-19, 227, 233-4, 241, 243, 244, 246, 248-9, 250-1, 254, 258

history $86-7$

original dances $14-15,87,88,97-9$, 126, 178-204, 220-1, 241, 248, 261

medal tests 50

men and dancing 13-14, 49, 110-11, $115,116-20,126-7$

Methodist Church 230

Merritt, Muriel 214

Metropolitan Police 11, 125, 129-30, 133

Meyrick, Kate 124, 131, 133-4,

Miami 159

Midnight Follies 66 
midway rhythm 72

Miles, Barbara 113

militant suffrage campaign 26

military 28, 55, 180, 194, 196

Second World War 208, 214, 215, 216, 217, 218, 219, 220, 221, $225,226-7,228-9,231$

Miller, Glenn 248

Mills, Annette 66, 201

Mills, Florence 199-200

Ministry of Labour 164

Ministry of Supply 217

Mississippi Delta 28

modernism 7, 20

modernity $20,82-3$

dancing as modern $12,30-5,39$, 118,252

modern ballroom dancing $2,7,10$, $11-12,14,15,21,29,30-35,37$, 38, 44, 45, 47, 54, 60, 113-14, $118,143,148,151,152,153$, 180, 181, 182, 189, 199-200, 242, 246, 247, 252-3, 254, 262, 263-4

see also English style of ballroom dancing

Modern Ballroom Dancing (Silvester) 53, 55

modern waltz 21, 29, 45, 48, 49, 51-2, 150, 157, 168, 172, 194, 198,262

see also standard four

modern woman 34, 107-14

monarchy 180, 196

monkey glide 23

moochi 72

Moore, Alex 55, 167, 200, 246-7, 249, 253,259

morale 209, 210, 212, 215, 216, 221, 223

Morris, Margaret 32

Mortimore, Philip 247

Morton, Jelly Roll 28

Mouvet, Maurice 48, 142, 151, 153, 163

Munich Crisis 182, 190, 191

musical theatre 21, 23, 24, 27, 169, 178-9 musicians $21,28,73,160,164,166$, 196, 221

see also dance bands

Musicians Union 164

music hall 4, 10, 50, 86, 143, 164, 185, $195,196,224$

music industry $7,71,78,100,143$, $164,167,170,180$

music publishing $11,21,62,70,94$, $180,181,193,243,258,264$

Mussolini, Benito 44, 190, 222, 223

Murrow, Edward R. 211-12, 225, 227

nation $4,9,10,12,45,136-7,144$, $160-3,201,253$

dancing and national characteristics $1,8,14,25,30,70,144-56$, $160-8,170-3,248,257-8,263$

dancing and national unity $33-4,143$, 144, 145-7, 209, 220, 225-7, 250

see also Anglicisation; commercial nationalism; Lambeth Walk as national dance; national identity National Association of Teachers of Dancing 52

national identity 3-6, 7, 39, 142-4, 149, 153, 171-3, 179-80, 184, 189-91, 196-7, 202, 209, 212, $234-235,241-2,248-50$

embodied through dancing 2, 4, 5, 9-10, 14-15, 143-4, 148-50, 160, 172-3, 188-90, 192, 209, 225-6, 236, 253-4, 262-3

see also nation

National Society of Amateur Dancers 52,166

National Vigilance Association 124, 134

Nava, Mica 16n.3, 83

Nazis 190-1, 222-5, 235, 254, 259

Newcastle 93, 226

New Orleans 28

Newton, Dorothy 55

Newton, Ramon 101, 154

New Woman 26

New York 21, 27, 28, 142, 144, 148, $159,163,164,167,171,182$

New Zealand 84

Nicolson, Juliet 20 
nightclubs 21, 94-5, 111, 121-2, 124-5, 129-30, 263

\section{Norway 222}

Nott, James 7, 82, 83, 100

Nottingham 82, 83, 87

novelty dances $4,5,173,178-204$, 219-21, 246, 261

Official Board of Ballroom Dancing 52, $55,60,71,135,159,168,259$

old-time dancing 22, 31, 91, 198

one-step 1, 21, 24, 26, 29, 49, 62, 84, $91,150,152,253,261$

see also standard four

Orientalism 80, 83-4

Original Dixieland Jazz Band 28-9, 80

Page, Anthony 194

palais de danse see dance halls

palais glide 183

palais stroll 179

Paramount dance hall 87, 91, 92, 195, 219, 233-4, 242-3, 244, 245, 246,251

Paris 1, 7, 8, 21, 22, 24, 27, 158,222

Park Parade 14, 179, 193-4, 196, 196-9, 202-3

party dances see novelty dances

Payne, Jack 101, 164, 184, 193

people's war 15, 190, 209, 225

Pearson, George E. 107-9

Peter Maurice Music Company 193, 248

Phoney War 212, 216, 241

piccolino 169

Pickford, Mary 80

Pierre, Monsieur 61, 117, 200, 201, 246

Plaza dance hall (Glasgow) 169

Plymouth 212

Polish pilots 227

polka 7, 21, 31, 198, 261

Popular Music and Dancing Weekly 99, 135

popular press $7,11,30,36,56,67,73$, 99-100, 110-11, 115, 117, 131,
$161,163,184,212-13,214,231$, 234, 243

Prince of Wales 67, 117

Princes Galleries 60

propaganda 209, 217

prostitution 122, 124, 128, 129-31,

132-3

provincial dancing 57, 93-4, 121, 145-7

Public Morality Council 124-5

Purcell, Florence 71

quadrille 21, 31, 181

Queen's Hall 51

quickstep 10, 45, 70-1, 91, 95-6, 157, 159, 168, 172, 244, 247

see also standard four

Quinn, James 199-200, 263

race $3,4,6,7,9,10,12,15,27,39$, 49, 84, 92, 121, 143, 153, 144, 161, 170, 180, 197, 203-4, 209, 225-6, 231, 241, 249-52, 254, 264

dancing and racial characteristics 8 , 148-51, 154-6, 171-2, 250-1

see also racial violence; racism; whiteness; whitening

racial violence $232-6$

racism 144, 164, 170, 226, 231-6

against dancing 2, 25, 37, 67, 151-4, 171-2, 197, 249-52

see also colour bars; racial violence radio $7,11,55,56,57,71,73,78$, 100-1, 114, 201, 217, 223, 258, 264

see also $\mathrm{BBC}$

ragtime dancing $12,20,21,22-4$, $25-26,53,147,152,252$

ragtime music $22-4$

rationing 214, 226, 227

Representation of the People Act (1918) 33,112

respectability $13-14,107,121,127$, $150,198,209,229,231,250$

and dance halls 2-3, 4, 79, 121-2,

$124-7,128,134,136,245$

restaurant dancing 94-5, 121 
Reynolds, David 228

Richardson, Philip J.S. 19-20, 21, 22, 25, 27, 31, 32, 35, 39n.6, 44, 46-9, 51, 53, 55, 57, 65, 67, 71, $142,144,146,148,156,159$, $161,168,182,183,187,188$, 201, 259

Ritz dance hall (Manchester) 86, 210, 218

Reiger, Bernhard 30

Rhodesia 159

Roberts, Robert 121

Robinson, Danielle 9, 153, 252

rock n' roll 15, 263

Rogers, Ginger 169-70, 172, 243

romance 4, 115, 122

Rose, Sonny 166

Rose, Sonya 228

Roy, Harry 102

Royal Air Force 224, 226-7, 231

Royal Albert Hall 68

Royal Family 80, 117, 178, 193, 261 see also Prince of Wales

Royal dance hall 92, 88, 130, 245

royal minuet 261

Royal Opera House 86, 87, 214, 246

Royal Navy 227

rumba $14,144,151,167-8,172$

Samuel, Raphael 189

Savoy Havana Band 101

Savoy hotel 25, 101, 154, 215, 219

Savoy Orpheans band 101

Scotland 6, 169, 188, 216

Scott, Edward 25, 151

Second World War 5, 6, 12, 15, 57, 86, 129, 136, 179, 180, 191, 194, 195, 208-36, 241-2, 247, 253-4, 258

sequence dancing 181, 182-3, 200, 201

Services Spotlight 217

Sevareid, Eric 212

sexuality 3, 14, 27, 39, 107, 115, 136, $145,209,225,228-32,236$

in dancing $2,25-6,143,144,150$, 153, 198, 245, 250, 257

and public dancing spaces 4, 122-3, 126-7
Sharp, Cecil 20, 31-2

Sheffield 87

Sherry's dance hall (Brighton) 86, 247

Sibley, Marie 132, 134

Sielle, Robert 63-4, 66

Singapore 221

Silvester, Victor 10, 32, 45, 49, 53, 55, 56, 66, 67, 101, 128, 130, 131, 146, 153, 154, 155-7, 158, 159, 166-7, 200, 208, 247-8, 249, $254,258,260,262$

Simmons, Murial 55

six-eight 72, 143, 163, 165

Spain 159

Sousa, John Philip 23

South Africa 51, 159, 258

South London Dance Association 126

Soviet Union 227

standardisation 3-4, 12-13, 35, 45-9, $51,53,55-6,60,64,68,72$, 96-7, 110, 142-52, 157, 160-1, $165,168,171,182,249$

standard five $65,69,165,183$

standard four 12, 49, 60, 71, 72, 95, 97, 144, 159, 166, 182, 200, 247. 259

see also foxtrot; modern waltz; one-step; quickstep; tango

Star 71, 100, 258

Stepping Out 224

Stewart, Maxwell 55, 115, 148, 150-1, 154, 155, 166

Stop Flirting 169

square dancing 261

Strictly Come Dancing 264

subscription dances 78-9

swing dancing 183, 243, 247

see also jitterbug; Lindy hop; truckin'

Swing Fever Jitterbug 146, 248, 249 , 250, 251, 252, 253

swing kids 254

swing music 96-7, 166, 170-1, 172, 203-4, 242, 254, 262

Switzerland 159

Sykes, Pat 55

tango $1,4,7,8,10,20,21,24-6,29$, $45,48,49,50,53,62,63-5,70$, 
$91,95-6,117,149-50,151,167$, 168,257

see also standard four

Taylor, Cecil 52, 55, 58, 77, 94, 117, 148, 156, 167, 186-7, 200, 246

Tebbutt, Melanie 6, 117, 120

television 55, 56, 73, 179, 204n.5, 258

theatre $7,50,106,210$

Thompson, Andrew 6

Tony's Ballroom (Birmingham) 93, 97

tourists $1,2,21,73,159,170$

Tower Ballroom 79

trebla 14, 72, 143, 161-3, 165, 198

trek (dance) 179

truckin' 14, 96-7, 144, 170-3, 242, 249,250

Tucker, Sherrie 242

turkey trot 23, 25

tuscana $220-1$

twist (dance) 15

Tynegate-Smith, Eve 44, 55, 58-9

United States 2, 6, 8, 25, 26, 51, 79, $96,147,155,157-8,159,164$, 170, 182, 184, 211, 227, 243, 244

influence on dancing $1-2,8,12,20$, 21, 22-4, 27, 30-1, 65, 66, 70, 73, 84, 142-4, 148, 150, 156, $159,160,163,166,167-8$, 170-3, 181, 183-5, 241-4, 246, 248-52, 253-4, 257, 259

see also African Americans; Americanisation

Variety Artists Federation 164 vaudeville 169

Walkowitz, Judith 6, 92

Walrond, Arthur 231, 232, 235

Walton, Florence 142, 153

waltz 1

Victorian 7, 21-2, 31, 35, 151, 261

see also Boston (dance); modern waltz

Warner, Phyllis 215, 225

war 3, 7, 182, 264

see also First World War; Second

World War

war veterans $19,36,107-10,112,118$ war work 214, 215, 217, 221, 226, 231

Weaver, Emily 229

Wellesley-Smith, Douglas 155-6

Wembley, Wibbly, Wobbly Dance 84

West End dance hall (Birmingham) 93

West Indies Calling 227

Wilson, Avice 232, 236

White, Louise 215

White, Walter 236

whiteness 143, 153, 252, 161, 172, $173,197,203-4,250,252$

whitening 26, 49, 143, 153-4, 203, 249-50

Wigley, Nelly 37

Wild West shows 8

Wimbledon 226

Wimbledon Palais 83, 85, 87

Winnipeg 159

women 4, 7, 116, 121, 264

and war 34-5, 209, 214-15, 217, 221, 227-9

women and dancing 2-3, 6, 13-14, $122,123,126-7$

Edwardian period 26, 150

interwar period 34-5, 49, 107-16, 127

Second World War 228-9, 231-3, 253

Women's Auxiliary Fire Service 227

Women's Voluntary Service 229

World Ballroom Dancing Congress 259

World Championship of Ballroom Dancing 55

World Dance Council 259

Yale blues 72

yam (dance) 98

Yorkshire 183, 185, 186

Young, Arthur 194

Young Women's Christian Association 113,116

youth $3,7,22,23,26,31,36,93,97$, 114, 117, 198, 241, 242, 247, $252,254,257$

youth culture 6

Zimring, Rishona 7, 20

Zweiniger-Bargielowska, Ina 37 